\title{
Spatial Coherence Patterns of Extreme Winter Precipitation In The United States
}

\author{
Abhirup Banerjee ( $\square$ abhirup.banerjee@pik-potsdam.de ) \\ Potsdam Institute for Climate Impact Research https://orcid.org/0000-0002-7101-0914 \\ Matthias Kemter \\ University of Potsdam \\ Bedartha Goswami \\ University of Tübingen \\ Bruno Merz \\ Helmholtz Centre Potsdam \\ Jürgen Kurths \\ Potsdam Institute for Climate Impact Research \\ Norbert Marwan \\ Potsdam Institute for Climate Impact Research
}

\section{Research Article}

Keywords:

Posted Date: January 17th, 2022

DOI: https://doi.org/10.21203/rs.3.rs-1243541/v1

License: (c) (i) This work is licensed under a Creative Commons Attribution 4.0 International License.

Read Full License 


\title{
Spatial coherence patterns of extreme winter precipitation in the United States
}

\author{
Abhirup Banerjee · Matthias Kemter - Bedartha Goswami · Bruno \\ Merz • Jürgen Kurths • Norbert Marwan
}

Received: date / Accepted: date

\begin{abstract}
Extreme precipitation events have a significant impact on life and property. The United States experiences huge economic losses due to severe floods caused by extreme precipitation. With the varied ter5 rain, it becomes increasingly important to understand the spatial variability of extreme precipitation to conduct a proper risk assessment of natural hazards such as floods. In this work, we use a complex network based approach to identify distinct regions exhibiting spa-
\end{abstract}

\footnotetext{
Abhirup Banerjee

Potsdam Institute for Climate Impact Research, Potsdam, Germany

Institute of Environmental Science and Geography, University of Potsdam, 14476 Potsdam, Germany E-mail: abhirup.banerjee@pik-potsdam.de

Matthias Kemter

Institute of Environmental Science and Geography, University of Potsdam, 14476 Potsdam, Germany

Helmholtz Centre Potsdam, GFZ German Research Centre for Geosciences, Potsdam, Germany

Potsdam Institute for Climate Impact Research, Potsdam, Germany

Bedartha Goswami

University of Tübingen, 72074 Tübingen, Germany

Bruno Merz

Helmholtz Centre Potsdam, GFZ German Research Centre for Geosciences, Potsdam, Germany

Institute of Environmental Science and Geography, University of Potsdam, 14476 Potsdam, Germany

Jürgen Kurths

Potsdam Institute for Climate Impact Research, Potsdam, Germany

Institute of Physics, Humboldt Universität zu Berlin, Germany

Norbert Marwan

Potsdam Institute for Climate Impact Research, Potsdam, Germany

Institute of Geoscience, University of Potsdam, 14476 Potsdam, Germany
}

tially coherent precipitation patterns due to various underlying climate mechanisms. To quantify interactions between event series of different locations, we use a nonlinear similarity measure, called the edit-distance method, which considers not only the occurrence of the extreme events but also their intensity while measuring similarity. Using network measures, namely, degree and betweenness centrality, we are able to identify the specific regions affected by landfall of atmospheric rivers in addition to those regions where the extreme precipitation due to storm track activity is modulated by different mountain ranges such as the Rockies and the Appalachians. Our approach provides a comprehensive picture of the spatial patterns of extreme winter precipitation in the United States due to various climate processes despite its vast complex topography.

\section{Introduction}

Extreme precipitation poses a serious threat to lives and livelihood of people all around the world. With the intensification of extreme precipitation and flood events over most climate regions (Tabari, 2020; Easterling et al, 2017; Janssen et al, 2014; Vu and Mishra, 2019; Kunkel et al, 2012) due to climate change, understanding the spatial variability of extreme precipitation is crucial to manage the big socioeconomic losses often associated with them (Merz et al, 2021). Previous studies have shown that extreme precipitation connectivity in the US is highest during the winter months (Touma et al, 2018), while river flood connectivity is higher in spring in the Rocky mountains and central US due to snow melt (Brunner et al, 2020). As reported in the billion-dollar weather and climate disasters catalog released by the NOAA/National Centers for Environmen- 
tal Information (NCEI), in the period 2010-2020, 328 people were killed due to flooding and winter storms

45 in the U.S., and more than $\$ 77$ billion (U.S. dollars) worth of economic damages were caused (Weather and for Environmental Information, NCEI). This included, for instance, the above-average precipitation leading to severe flooding in the Mississippi and Missouri Rivers and their tributaries during the winter season of 2019 (December 2018 - February 2019) (Hoell et al, 2021; Flanagan et al, 2020). Thus our study focuses on extreme precipitation in the winter months (DJF), during which rainfall can cause floods directly and snowfall

55 leads to the accumulation of snow packs for the melting season.

Numerous studies have analyzed extreme precipitation events across the United States (Mondal et al, 2020; Najibi et al, 2020), meteorological causes of secu-

60 lar variations (Kunkel et al, 2012), spatiotemporal variability (Kursinski and Mullen, 2008), and their relation to large-scale meteorological patterns (Agel et al, 2019). Here, we focus on the spatial connectivity of extreme precipitation events, which is relevant for river flood generation and the spatial extent of simultaneous flooding (Brunner et al, 2020; Kemter et al, 2020). Understanding the spatial dependence of extreme precipitation and its underlying mechanism is important to assess risk from natural hazards. Simultaneous extreme

70 precipitation across large scales can lead to synchronous flooding in multiple states, which has a greater societal and financial impact than independent, localized flood events due to regional interdependencies in risk management, infrastructure, and insurance (Jongman et al, 75 2014).

We use a complex network based approach to study spatial patterns of extreme winter precipitation in the United States. Climate network analysis can help to identify the regions which are most likely to experience

80 concurrent precipitation extremes and which climatic conditions are responsible for their generation. The climate network belongs to the category of functional network, i.e., in which pairwise dependency of station or grid data is computed, and the network topology is rep85 resented by different network measures (Donges et al, 2009; Fan et al, 2021; Tsonis and Roebber, 2004). The network representation of climate data allows us to study pairwise interactions between climate variables of different locations. However, standard similarity measures, 90 such as Pearson or Spearman correlation coefficients are not suitable to evaluate the relationship within extreme precipitation data which are event-like time series. In the past decade, a specific and nonlinear synchronization measure, in particular event synchronization (ES) (Quian Quiroga et al, 2002) have been used extensively to construct climate networks for event-like data such as extreme precipitation (Malik et al, 2011; Stolbova et al, 2014; Ozturk et al, 2019), heat wave pattern (Mondal and Mishra, 2021) etc. Boers et al (2013, 2014a,b) used complex networks constructed based on ES to study the South American Monsoon and reveal the global extreme precipitation pattern (Boers et al, 2019). Konapala and Mishra (2017) used the same climate network framework to study hydroclimatic extreme events. Agarwal et al (2017) introduced multiscale event synchronization by combining wavelet transform and $\mathrm{ES}$.

However, ES only considers the time of occurrence of events to identify the events coincidence and use this as a measure for similarity, but not the difference in strength or amplitude of the events. While very few previous works (Ciemer et al, 2018) have proposed some modified correlation measures to investigate spatial covariability pattern of general precipitation (also considering the amplitude variability), these methods are linear and, thus, not suitable to study extreme precipitation behaviour.

In our study, we use a special distance metric, particularly designed to study the similarity between spike trains, called edit distance (ED), first proposed by Victor and Purpura (1997) and later extended by Hirata and Aihara (2009). This metric has been used in combination with recurrence plot to analyze the recurrence property of marked point process data (Suzuki et al, 2010), paleoclimate data (Ozken et al, 2015, 2018), and extreme event-like hydrological data (Banerjee et al, 2021). Recently, Agarwal et al., (accepted) also applied this measure to study the extreme rainfall pattern in the Ganga River basin. Under this framework, we consider here each event series as a marked point process and measure the similarity between two such event series by optimizing the cost of transformation associated with transforming one event series to another one through elementary operations, such as shifting, addition or deletion of events.

Spatial patterns of different network measures, namely degree and betweenness centrality are used to study the spatial connectivity of extreme winter (DecemberJanuary-February (DJF)) precipitation events. While the degree field is based on local topological informa- ${ }_{140}$ tion, the path-based betweenness centrality field includes global topological information (Donges et al, 2009). Through our approach, we are not only able to identify regions with distinct extreme precipitation patterns, but also delineate the regions affected by atmospheric rivers and tornadoes. The patterns of Sec. 2, we describe in detail, the data and the methodology. In Sec. 3, we draw an in- 
terpretation based on our network analysis and discuss the results from a climatological point of view.

\section{Data and Methodology}

\subsection{Data source and data pre-processing}

In this study, we use daily averaged precipitation, geopotential height, wind at different pressure levels, and vertically integrated water vapour (IVT) flux data dethe period $1980-2020$. The spatial resolution used is $0.5^{\circ} \times 0.5^{\circ}$. It is worth mentioning here that although reanalysis precipitation data do show biases compared to the observations, observational datasets typically ei-

160 ther have a limited spatial coverage (GPCC, TRMM, etc.), lower resolution (GPCP) or a limited temporal coverage (TRMM). The ERA5 shows in most cases, smaller biases than other reanalysis datasets (JRA-55, MERAA-2)(Hassler and Lauer, 2021). However, we verify the robustness of our results by comparing them with those obtained using JRA-55 (Japan Meteorological Agency, Japan, 2013) (see figures in the Supporting information).

\subsection{Network construction}

170 A network or graph comprises two main components: a set of nodes $V$ and a collection of edges $E$. Mathematically, a network is expressed as $G=\{V, E\}$ (Sivakumar and Woldemeskel, 2014; Donges et al, 2009). In case of climate network, each geographical grid point of the climate dataset is considered as a node and an edge is placed when there is statistically significant association or functional dependency between two nodes. To construct the climate network for extreme precipitation, first we transform the precipitation time series data at 180 each grid point into an extreme precipitation event series by considering those days as events for which precipitation is among the highest $5 \%$ of all values, including dry days without precipitation, in a particular season at that location, resulting in 4 to 5 events for bova et al, 2014). Here, we construct the network for extreme precipitation events to study its pattern of spatial variability.

In this study, we use the edit distance (ED) method, 190 which takes into account both the sequence and amplitude of events. In general, ED is a distance measure to quantify the similarity/ dissimilarity between two spike trains (Victor and Purpura, 1997; Banerjee et al, 2021). Additionally, ED considers each event series as a marked point process (Suzuki et al, 2010; Ozken et al, 2015, 2018). The idea is to transform an event series into another series by performing some elementary operations: shifting in time, amplitude modulation, and deletion/insertion of events (Fig. 1). A specific cost is assigned to each operation. The total cost of transformation from one event series to the other is computed by tracing the minimal-cost path.

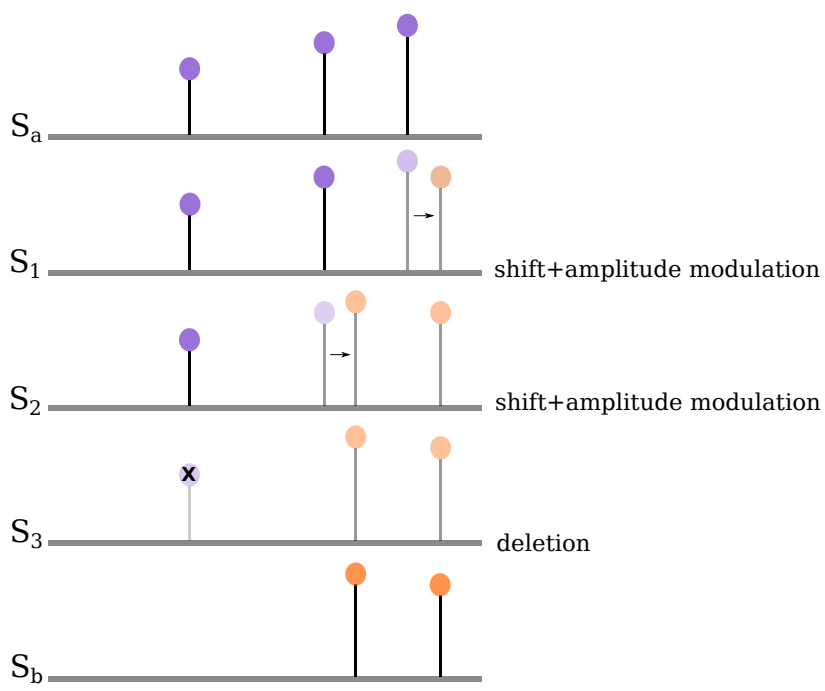

Fig. 1 Schematic of the transformation of segment $S_{a}$ to $S_{b}$ through four steps numbered as steps $S_{1}, \ldots, S_{3}$. The path shown is a minimal-cost path and all steps are elementary steps, i.e., shifting an event, amplitude modulation, deleting/inserting.

The mathematical formulation of the distance measure is described as follows. Consider two given segments $S_{a}$ and $S_{b}$, the minimum cost of transformation is defined as

$$
\begin{array}{r}
D\left(S_{a}, S_{b}\right)=\min _{C}\left\{\sum_{(\alpha, \beta) \in C} \Lambda_{0}\left\|t_{a}(\alpha)-t_{b}(\beta)\right\|\right. \\
\left.\quad+\Lambda_{1}\left\|L_{a}(\alpha)-L_{b}(\beta)\right\|\right\} \\
\left.+\Lambda_{s}(|I|+|J|-2|C|)\right\} .
\end{array}
$$

The time and amplitude of events are denoted as $t_{a}(\alpha)$, $t_{b}(\beta)$, and $L_{a}(\alpha), L_{b}(\beta), \Lambda_{0}$ and $\Lambda_{1}$ are the coefficient of cost of shifting in time and amplitude change. The first term of Eq. (1) sums the cost of shifting in time and amplitude change between the $\alpha^{\text {th }}$ event in $S_{a}$ and $\beta^{\text {th }}$ event in $S_{b}, C$ contains all the pairs associated in this operation. The second term in Eq. (1) denotes the deletion/ insertion operation, $|I|,|J|$ are the sets indices of events in $S_{a}$ and $S_{b}, \Lambda_{s}$ is the coefficient of cost of deletion. 
The minimum cost implies the highest similarity and vice-versa. We then calculate the transformation cost for every pair of event series $i$ and $j$ of the gridded extreme event dataset using the above method, which gives us the similarity matrix $Q_{i j}$ (here, cost matrix). Thereafter, we obtain the adjacency matrix $A_{i j}$ by thresholding the similarity matrix $Q_{i j}$ with a suitable threshold, which gives the edges of our network. below a certain th old, and $\delta_{i j}$ is the Kronecker delta to remove self loops. In the case of $\mathrm{ED}$, lower transformation cost between of grid cells whose value of the transformation cost is below the threshold $\epsilon$ will be connected by an edge. In this study, to find the significant edges, we fix the edge density of the network at $\rho=\frac{2 E}{N(N-1)}=5 \%$ and 235 choose the corresponding threshold $\epsilon(\rho)$ (Malik et al, 2011; Stolbova et al, 2014; Wiedermann et al, 2017).

\subsection{Network measures}

Various network measures are used to quantify the network topology which provide novel insights into the underlying dynamics of the system over different spatial scales (Donges et al, 2009). We use two network measures to quantify and characterize the spatial pattern of extreme precipitation. One of the simplest local network measure is the degree which measures the centrality of a node based on how well-connected it is. The Degree $k_{i}$ of a node $i$ is defined as

$k_{i}=\sum_{j=1}^{N} A_{i j}$

where $N$ is the total number of grid points (nodes). It quantifies the number of direct connections node $i$ mate networks, nodes with higher degree values $k_{i}$ indicate the spatial distribution of similar variability, related to linked processes (Boers et al, 2014b), such as large-scale atmospheric circulation (Malik et al, 2011;

245 Boers et al, 2013, 2014b). It has been used to identify the highly connected geographical sites (super-nodes) and their association with atmospheric teleconnection pattern (Tsonis et al, 2008; Radebach et al, 2013; Agarwal et al, 2019).

The second network measure we use is the betweenness centrality, which provides information about the global topology on the basis of shortest paths between pairs of nodes (Donges et al, 2009). Betweenness centrality $B C_{i}$ measures how much a node $i$ falls 'in between' two nodes in the network, i.e., acts as a bridge connecting two other nodes (Newman, 2010; Freeman, 1978). A node may not be well-connected (i.e., has low degree) but can be crucial to connect different parts of the network (Golbeck, 2015) (Fig. 2b). Betweenness is quantified by measuring the percentage of the shortest paths that must go through this specific node $i$ and is defined as

$B C_{i}=\sum_{j, k \neq i}^{N} \frac{\sigma_{j k}(i)}{\sigma_{j k}}$

where $\sigma_{j k}$ is the total number of shortest path between node $j$ and $k$ and $\sigma_{j k}(i)$ is the number of shortest paths that go via node $i$. In case of social network, BC indicates the importance of a node in controlling the flow of information in the network. However, for functional networks, such as climate networks, it represent boundaries between highly connected regions (Molkenthin et al, 2014; Tupikina et al, 2016). BC has been used to uncover energy flow patterns in the atmosphere (Donges et al, 2009) and has also been successfully applied to study the extreme precipitation patterns of different monsoon systems (Boers et al, 2013; Stolbova et al, 2014).

Correction for spatial embedding: When we choose a particular study area, we impose an artificial boundary in space. These boundaries influence the climate network (Rheinwalt et al, 2012; Boers et al, 2013) by cutting links that actually connect nodes with outer regions, hence affecting the network measures. Here we adopt the boundary correction procedure suggested by (Rheinwalt et al, 2012) as follows: We first generate 500 spatially embedded random networks (Barnett et al, 2007) (SERN) which preserve both the node position and the distribution of the spatial link lengths of the original network. After that, we compute the network measures for all SERN surrogates. The boundary-corrected ${ }_{75}$ network measure is obtained by dividing the original measure with the average of the SERN surrogates measure.

\section{Results and discussion}

\subsection{Calculation and network interpretation}

In this section, we analyze the winter extreme precipitation pattern using the above introduced complex network measures. Our climate network, constructed using the ED metric (mentioned in 2.2), considers both the 5 


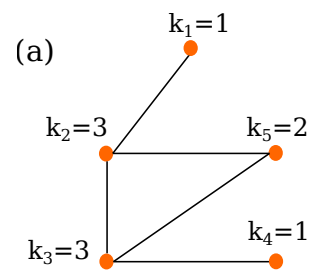

(b)

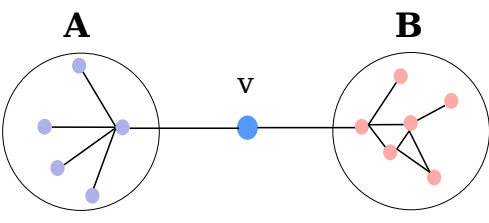

Fig. 2 Network measures: (a) Degree $k_{i}$ of the network nodes, based on the number of connections of node $i$ with other nodes. Degree measures how well-connected a node is in the network. (b) Betweenness centrality $B C_{i}$ of network nodes. Node $v$ has low degree but high betweenness because it acts as a bridge joining two groups of nodes $A$ ans $B$.

(a)

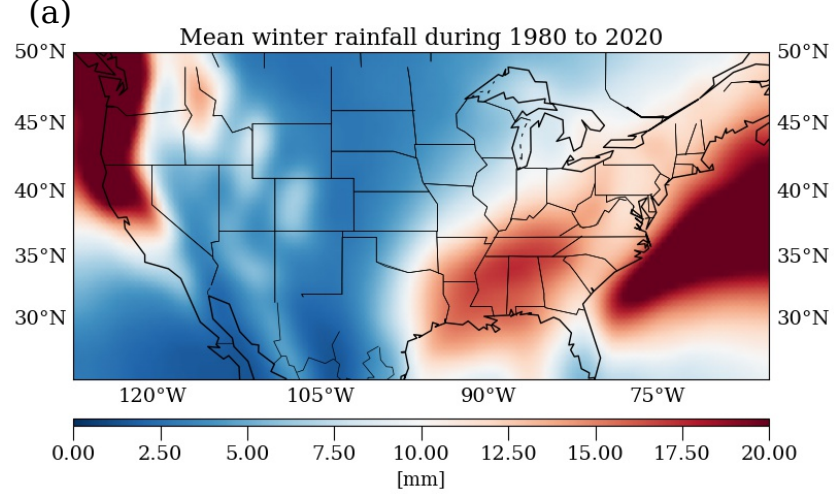

(b)

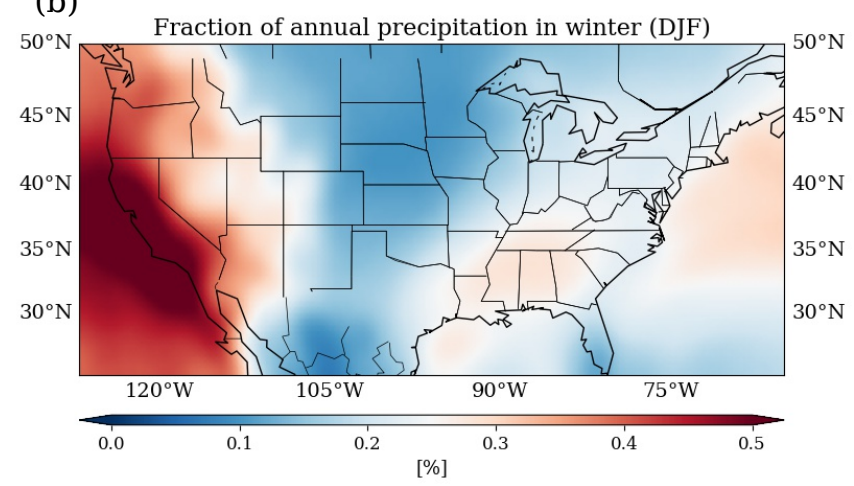

Fig. 3 (a) Mean daily winter precipitation from 1980 - 2020 (b) Mean winter precipitation anomaly as the fraction of mean annual precipitation falling in winter (same period) for ERA5 reanalysis data. Anomalies are highly positive along the West Coast and slightly positive along the southern flank of the Appalachians. Highly negative anomalies exist in the central north.

sequence and the amplitude of events when quantifying similarity. High degree values (Eq. 2) represent high connectivity of extreme precipitation events, i.e., grid cells having similar variation in extreme precipitation occurrence and intensity. We find in the corresponding network a relatively low degree in the northwestern part of the U.S. (Fig. 4a), suggesting less similarity of extreme precipitation behaviour with any other regions. A high degree is observed in the eastern Pacific Ocean and southwestern part of the U.S. To understand the connectivity pattern for these regions, we choose really small boxes A (low degree) and $\mathbf{B}$ (high degree), in the northwestern part of the U.S. and in the eastern Pacific Ocean respectively, and determine the number of links connecting these boxes with other nodes in the network (Figs. 5a,b). We find that connections with the region $\mathbf{A}$ are confined to a very small region centred more towards the coast, indicating a quite narrow corridor of moisture transport as typical for atmospheric rivers (Dettinger, 2013; Xiong and Ren, 2021; Hu et al, 2017; Gonzales et al, 2019). On the other hand, the connectivity of region $\mathbf{B}$ spans over a larger area in the Pacific Ocean and extending up to some parts of the southwestern coast. Such extended connectivity represents the impact region of a larger atmospheric pattern, such as tropical cyclones which are typical in this region to bring enhanced rainfall (Woodruff et al, 2013). We also observe high degree values in the Great Plains and northeastern parts of the U.S. Here, we choose another small box $\mathbf{C}$ which lies roughly in the Mississippi river watershed where a relatively high degree is observed (Fig. 5c). The connectivity pattern of this region shows a similar behaviour in extreme precipitation along the southwest-northeast direction. It should be noted that high elevation regions such as the Cascades, some parts of the Rockies and the Appalachians show relatively lower degree than low elevation region which was also observed by Agarwal et al. (2021) (accepted) in case of extreme precipitation networks constructed using editdistance. Similar observations are made in the results obtained using JRA-55 dataset (see Fig. S2a). Next we study the spatial patterns of BC (Fig. 4b), representing some striking structures revealing the transition zones between different atmospheric flows (Molkenthin et al, 2014; Tupikina et al, 2016) during winter in the U.S. Along the northwestern coast of the U.S., we find high betweenness but low degree. This implies that although these are relatively small regions of similar precipitation dynamics, they are transition zones of different atmospheric flow directions (Molkenthin et al, 2014), possibly because of spatial confinement and orographic lift due to the presence of topographical features such as 

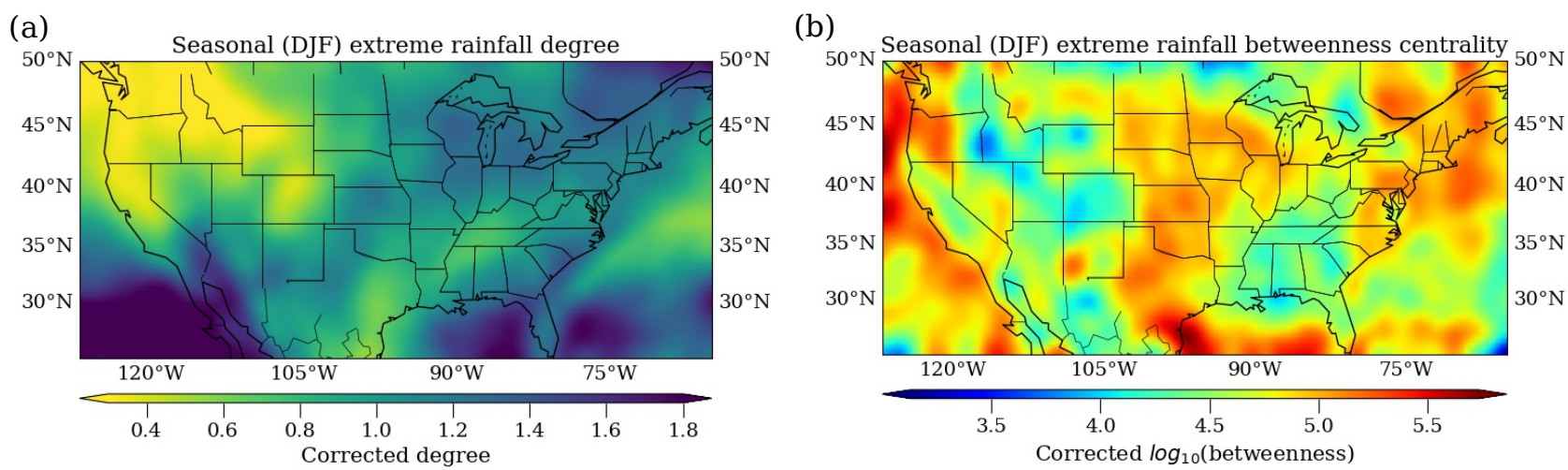

Fig. 4 (a) Degree, and (b) betweenness centrality for extreme winter (DJF) precipitation from 1980 to 2020.

mountains. The BC values are seen to continue downwards along the entire western coast, lining the land-sea boundary. The results obtained from ERA5 which is in contrast to those obtained from JRA-55, where they decrease substantially beyond $30^{\circ} \mathrm{N}$ southwards. We observe high BC values in the central U.S., i.e., from Texas towards the Midwest area, and in the northeastern region, which are also regions of high degree. This implies that while the lower elevation regions, east of the Rocky mountains (Great Plains) and the Appalachians (Coastal Plains), are large regions of spatially coherent extreme precipitation dynamics, big rivers and mountain features cause diversification of atmospheric flow

350 leading to different and strongly fragmented precipitation patterns. These observations are mostly similar with those seen in the network constructed using the JRA-55 dataset (Fig. S2b) except for the small disparity in $\mathrm{BC}$ values seen along the southwest coast. This cipitation data in the Pacific ocean close to the tropics (Hassler and Lauer, 2021).

\subsection{Climatological interpretation}

The low connectivity in the northwestern part of the and Rocky Mountains on precipitation. Precipitation gets "trapped" west of these ranges, and, thus, unconnected to the rest of the country, lowering the overall degree. In the high elevations, extreme precipita365 tion requires different conditions than at the coast, so the northwest coast and the mountain ranges are also not connected. However, as the rainstorms can travel more freely through the plains on the eastern side of the mountains, it leads to a higher regional similarity. The to extreme precipitation resulting in high $\mathrm{BC}$ values little inland up the northwest coast (Fig. 4b).

The southwestern part of the U.S. along with adjacent regions of the eastern Pacific Ocean exhibit a high connectivity due to a high fraction of winter precipitation despite a low mean winter precipitation (Fig. 3). This effect can be explained by the finding that this part of the eastern Pacific is a separate, relatively small and well-organized precipitation system (Zhang and Wang, 2021) as also seen from Fig. 5b. Elevation and slopes are much lower here than further north, so rainstorms can penetrate further into the land and cause nearsimultaneous precipitation along the land terrain.

The southwestern coast has high $\mathrm{BC}$ values similar to the northwestern coast indicating that they may be related to transition in opposing atmospheric flow direction. The western coast of the U.S. experiences heavy precipitation, and hence extreme streamflows, due to the ARs which contribute $30 \%$ to $45 \%$ of total winter precipitation (Dettinger, 2013; Xiong and Ren, 2021; $\mathrm{Hu}$ et al, 2017). ARs are relatively narrow filamentshaped conduits of moisture in the atmosphere transported from the lower latitudes to the mid and high latitudes (Gimeno et al, 2016; Guan and Waliser, 2015; Ralph et al, 2019). The activity of ARs starts during autumn and tends to shift southward along the Pacific coast later during the winter (Gonzales et al, 2019). However, these ARs may be associated to different regimes of large-scale Rossby wave breaking (RWB) - anticyclonic wave breaking (AWB) in the northwest and cyclonic wave breaking (CWB) in the southwest $(\mathrm{Hu}$ et al, 2017)(Fig. 6a,b). High BC values penetrate farther inland (Fig. 4b), in the northwest U.S., close to the western slope of the Cascades. This may be related to the AWB-ARs which arrive more orthogonally to the western Cascades due to their westerly impinging angle transforming moisture to precipitation due to orographic lift. On the other hand, the CWB-ARs have impinging angles which is more southwesterly, and there- 
(a)

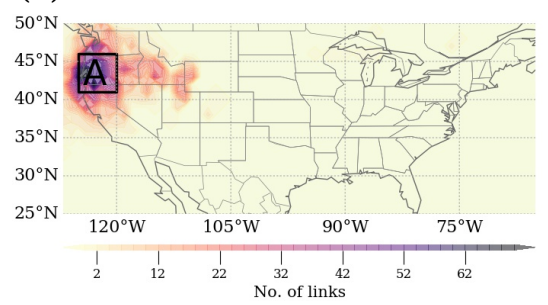

(b)

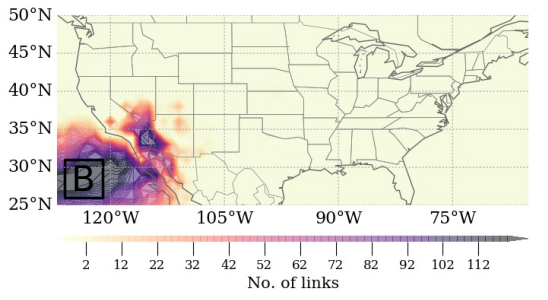

(c)

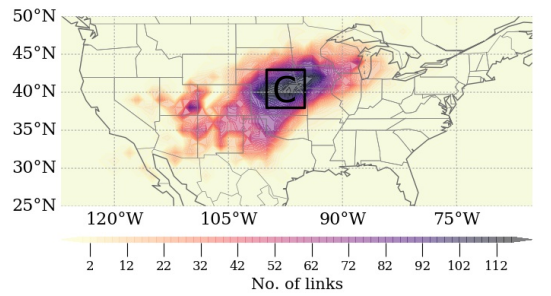

Fig. 5 Partial degree, i.e., the number links connected to the selected regions in the north-western United States (Box A), eastern Pacific Ocean (Box B), and in the central United States (Box C).

fore arrive more orthogonally to the east-west oriented Olympics in the northwest U.S. and the

northwest-southeast oriented Sierra Nevada along the southwest coast. Consequently, they causing intense precipitation along the western coast. The transformation of water vapour to extreme precipitation through the orographic lift (Barlow et al, 2019) albeit due to different regimes of RWB explains the high $\mathrm{BC}$ along the western coast. The relatively high degree in the southshorter track ARs close to central and southern California. The seasonal progression of the mean latitude position of the AR tracks southwards could also possibly explain the high $\mathrm{BC}$ values in this region (Gonzales 25 et al, 2019).

The southwest-northeast (SW-NE) inclination in connectivity of the high degree regions in the northeast U.S. and the Great Plains is in agreement with Najibi et al (2020) who found high similarity in anomalous extreme precipitation in winter in these regions. The eastern side of the Rockies also have high BC values which may be attributed to the pressure gradient seen in the atmosphere (Figs. 6a,b,c) (Molkenthin et al, 2014). The area roughly coincides with the loosely-defined region called the Tornado Alley, where tornadoes occur very frequently (Concannon et al, 2000; Bluestein, 2006). We also see a propagation of wind in the southwesterly direction in all atmospheric levels (Figs. 6a,b,c). The IVT seasonal composite anomalies (Fig. 6d) also show an 440 anomalously high moisture transport in this direction. This flow pattern is modulated by the presence of the Rocky mountains (Lukens et al, 2018) which suppress the storm-track activity by deflecting the westerly flow over land (Chang, 2009). This leads to a SW-NE tilt in the upper tropospheric jet (Fig. 6a) subsequently causing a downstream flow and hence high betweenness along those nodes.

High BC values along the northeast coast of U.S. may also be associated with high baroclinic instability formed due to the large land-sea temperature gradient in winter over northeastern U.S.(Brayshaw et al,
2009) which leads to an intensification of extratropical storms on the leeward side of the Appalachian mountains (Colucci, 1976; Lukens et al, 2018). Extreme precipitation in this region is mainly related to an anomalously high upward lift of air along the coast due to high vorticity advection, frequent warm conveyor belts and diabatic heating (Agel et al, 2019). The wind flow (Figs. 6a,b,c) and high anomalous IVT (Fig. 6d) along the northeast coast, leads to synchronous extreme precipitation in the region and hence high degree.

\section{Conclusions}

The climate network approach has been proven to be a robust and promising framework for studying various climate extremes such as extreme monsoon precipitation (Malik et al, 2011; Boers et al, 2013, 2014b), the influence of El Niño (Boers et al, 2014a), cyclone tracks (Gupta et al, 2021) etc. In this work, we studied the the spatial variability of extreme precipitation during winter for the United States, which has a very complex topography. For this, we employ the edit distance metric to measure pairwise similarity between extreme precipitation time series of different locations. Most of the earlier developed methods (Malik et al, 2011; Stolbova et al, 2014; Boers et al, 2013, 2014a; Wolf et al, 2020) consider only the timing of events when studying the similarities in event-like data. However, the edit distance emerges as a powerful alternative measure because it considers amplitude or strength of extreme events along with their time of occurrences when calculating the similarity.

Extension of the coherent regions depend on the orography, climatological season and the atmospheric circulations. An understanding of the spatial extent of regions of coherent extreme precipitation is necessary for risk assessment of natural hazards. Through a combination of network measures, viz., degree and betweenness centrality, we were able to identify the different regions of the U.S.which exhibit distinctly different extreme precipitation dynamics. While analyzing spatial 

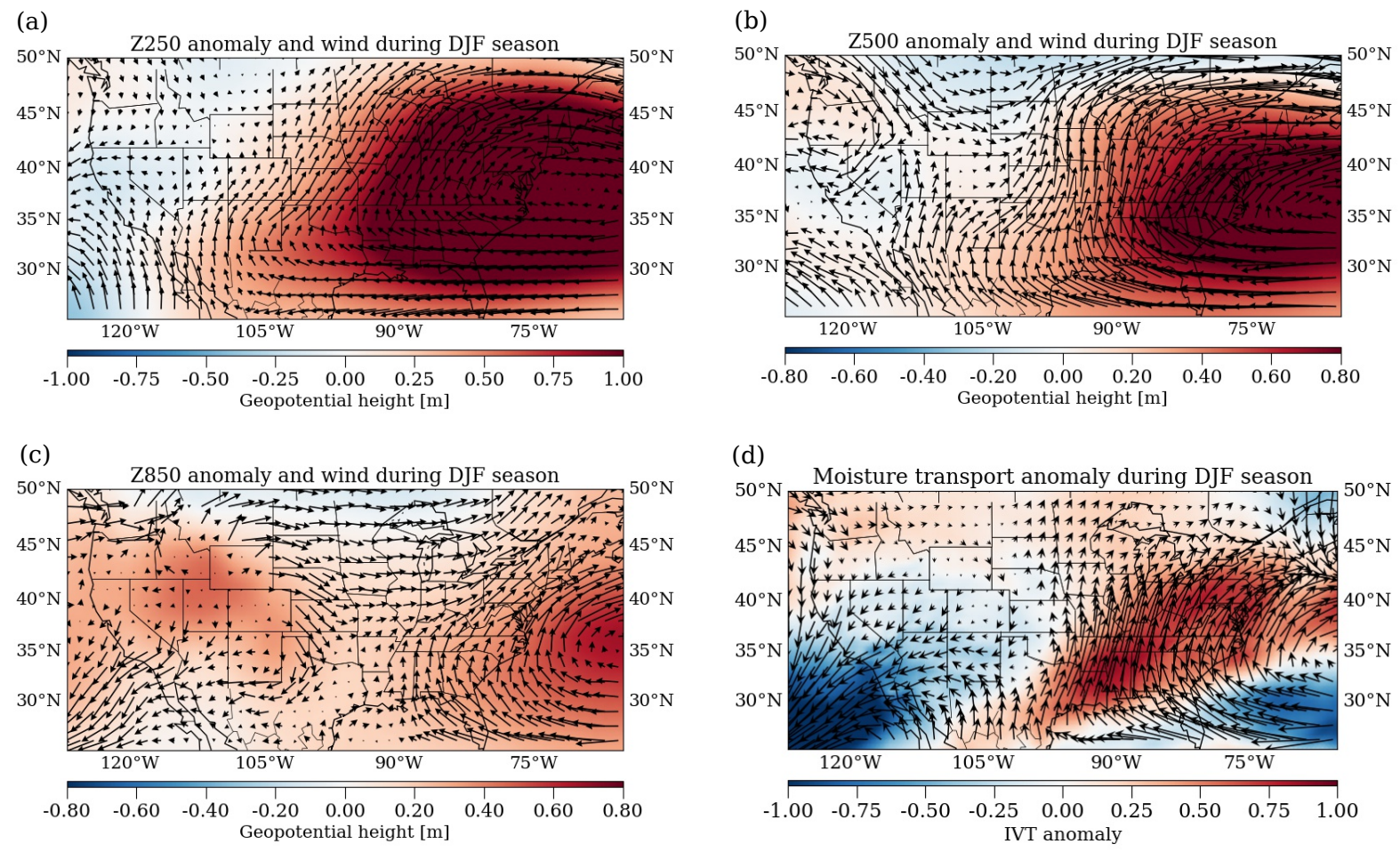

Fig. 6 (a)-(c) Geopotential height and wind in $250 \mathrm{hPa}, 500 \mathrm{hPa}$, and $850 \mathrm{hPa}$ atmospheric level, (d) vertically integrated water vapour flux anomaly during winter season (DJF).

patterns of degree differentiated between the northwest and the southwest coast on the basis of associated largescale atmospheric circulation, the high betweenness along the entire western coast brought to light the role of ARs and that of topographic barriers in causing extreme precipitation. The network measures also roughly identify the "Tornado Alley" (Concannon et al, 2000; Bluestein, 2006) region in the Great Plains where tornadoes are more frequent. The high degree pattern captured the 500 southwest-northeast (SW-NE) inclination (Najibi et al, 2020; Lukens et al, 2018) of extreme precipitation due to modulation of storms by the Rocky mountains. Similarly, a modulation of extreme precipitation due to other high ranges, such as the western Cascades and the Appalachians in the east of the country, were also reflected in the network connectivity.

Our complex network based approach provides a comprehensive overview of the distinct regions which experience spatially coherent extreme winter precipitation in the United States albeit due to various climate processes. The similarity measure used in this study, the edit distance, comes out as a very promising alternative to study extreme precipitation patterns in regions exhibiting very intricate climate variability such as the US. Future work may include further refinement of the method by incorporating more sophisticated statistical significance tests. The method can also be applied to study the effects of increasing intensity of extreme precipitation for different large-scale monsoon systems and to possibly identify teleconnections.

\section{Supplementary}

Corresponding author Abhirup Banerjee, Email: abhirup.banerjee@pik-potsdam.de

Acknowledgements This research has been funded by the Deutsche Forschungsgemeinschaft (DFG) within graduate research training group GRK 2043/1 "Natural risk in a changing world (NatRiskChange)" at the University of Potsdam. $\mathrm{AB}$ would like to thank Shraddha Gupta and Zhen Su for valuable discussion.

\section{Author Declarations}

- Conflict of interests The authors declare no competing interests.

- Consent to participate Not applicable.

- Data availability The data/reanalysis that supports the findings of this study are publicly available online: ERA5 Reanalysis data (Hersbach et al, 2020), https://cds . climate. copernicus.eu/ and JRA-55 (Japan Meteorological Agency, Japan, 2013) reanalysis data.

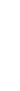


(a)

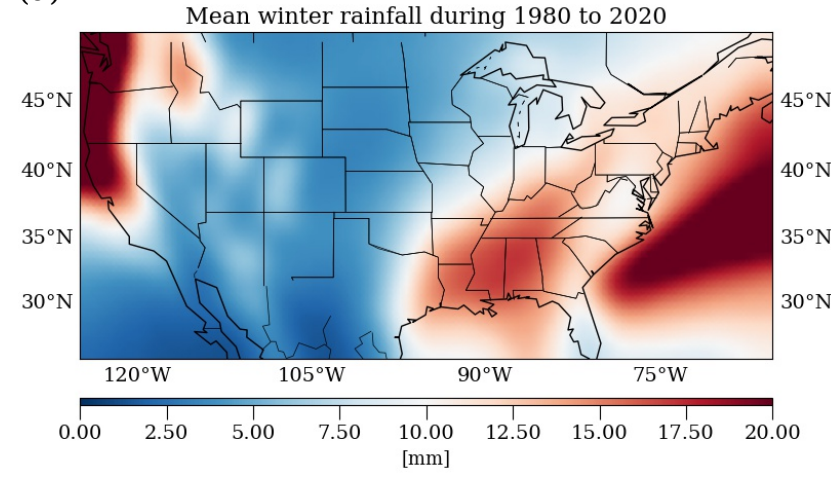

(b)

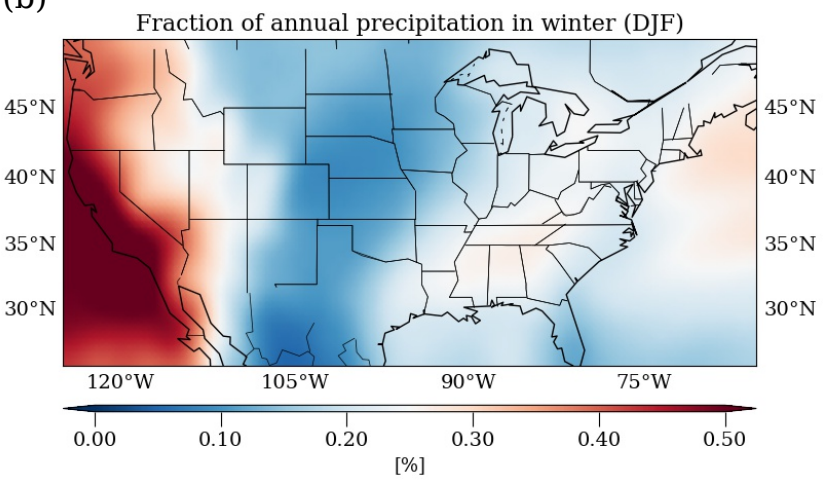

Fig. S1 (a) Mean daily winter precipitation from 1980 - 2020 (b) Mean winter precipitation anomaly as the fraction of mean annual precipitation falling in winter (same period) for JRA-55 data.

(a)

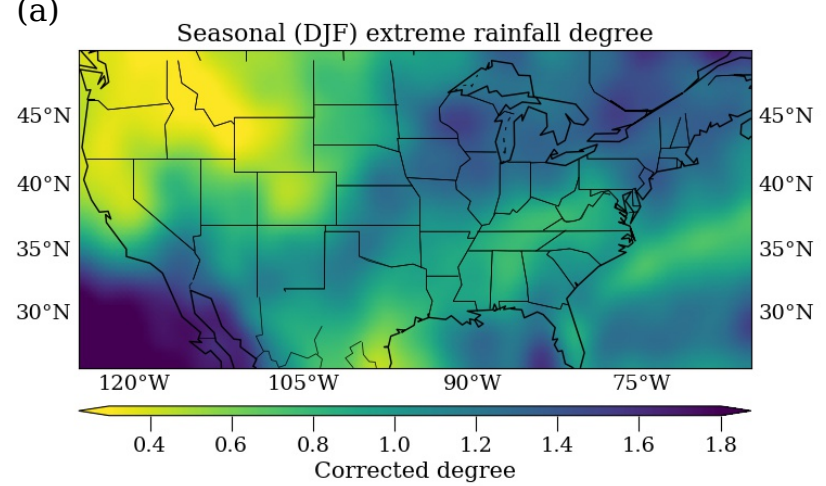

(b)

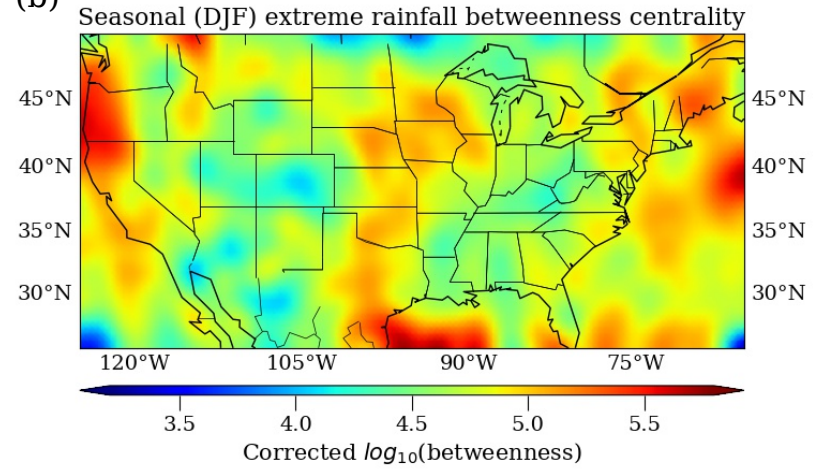

Fig. S2 (a) Degree, and (b) betweenness centrality for extreme winter (DJF) precipitation from 1980 to 2020 using JRA 55 reanalysis data.

- Code availability The readers are requested to contact the corresponding author Abhirup Banejee regarding code and analysis.

- Author's contribution AB, NM, developed the theoretical formalism. AB carried out the experiment. MK and BG helped with result interpretation. BM and JK closely supervised the work. $\mathrm{AB}$ took the lead in writing the manuscript. All authors provided critical feedback and helped shape the research, analysis and manuscript.

\section{References}

Tabari H (2020) Climate change impact on flood and extreme precipitation increases with water availability. Scientific Reports 10

Easterling DR, Kunkel KE, Arnold JR, Knutson T,
Janssen E, Wuebbles DJ, Kunkel KE, Olsen SC, Goodman A (2014) Observational- and model-based trends and projections of extreme precipitation over the contiguous united states. Earth's Future 2(2):99113, DOI https://doi.org/10.1002/2013EF000185, URL https://agupubs onlinelibrary. wiley.com/doi/abs/10.1002/2013EF000185, https://agupubs.onlinelibrary.wiley.com/ doi/pdf/10.1002/2013EF000185

Vu TM, Mishra AK (2019) Nonstationary frequency analysis of the recent extreme precipitation events in the united states. Journal of Hydrology 575:9991010, DOI https://doi.org/10.1016/j.jhydrol.2019. 05.090, URL https://www.sciencedirect.com/ science/article/pii/S0022169419305359

Kunkel KE, Easterling DR, Kristovich DAR, Gleason B, Stoecker L, Smith R (2012) Meteorological causes of the secular variations in observed extreme precipitation events for the conterminous united states. Journal of Hydrometeorology 13(3):1131 - 1141, DOI 10.1175/JHM-D-11-0108.1, URL https://journals.ametsoc.org/view/journals/ hydr/13/3/jhm-d-11-0108_1.xml 
Merz B, Blöschl G, Vorogushyn S, Dottori F, Aerts J, Bates P, Bertola M, Kemter M, Kreibich H, Lall U, Macdonald E (2021) Causes, impacts and patterns of disastrous river floods. Nature Reviews Earth \& Environment 2:1-18, DOI 10.1038/s43017-021-00195-3

Touma D, Michalak AM, Swain DL, Diffenbaugh NS (2018) Characterizing the spatial scales of extreme daily precipitation in the united states. Journal of Climate 31(19):8023 - 8037, DOI 10.1175/JCLI-D-18-0019.1, URL https://journals.ametsoc.org/view/journals/ clim/31/19/jcli-d-18-0019.1.xml the American Meteorological Society 101(6):E817

Flanagan PX, Mahmood R, Umphlett NA, Haacker
E, Ray C, Sorensen W, Shulski M, Stiles CJ,
doi/abs/10.1029/2020GL088185, e2020GL088185 2020GL088185, https://agupubs . onlinelibrary . wiley.com/doi/pdf/10.1029/2020GL088185

Najibi N, Mazor A, Devineni N, Mossel C, Booth JF (2020) Understanding the spatial organization of simultaneous heavy precipitation events over the conterminous united states. Journal of Geophysical Research: Atmospheres 125(23):e2020JD033,036, DOI https://doi.org/10.1029/2020JD033036, wiley.com/doi/abs/10.1029/2020JD033036, e2020JD033036 10.1029/2020JD033036, https : //agupubs.onlinelibrary.wiley.com/doi/pdf / 10.1029/2020JD033036

Kursinski AL, Mullen SL (2008) Spatiotemporal variability of hourly precipitation over the eastern contiguous united states from stage iv multisensor analyses. Journal of Hydrometeorology 9(1):3 - 21, DOI 10.1175/2007JHM856.1, URL https://journals.ametsoc.org/view/journals/ hydr/9/1/2007jhm856_1.xml

Agel L, Barlow M, Colby F, Binder H, Catto J, Hoell A, Cohen J (2019) Dynamical analysis of extreme precipitation in the us northeast based on large-scale meteorological patterns. Climate Dynamics 52:1-22, DOI $10.1007 / \mathrm{s} 00382-018-4223-2$

Kemter M, Merz B, Marwan N, Vorogushyn S, Blöschl G (2020) Joint Trends in Flood Magnitudes and Spatial Extents Across Europe. Geophysical Research Letters 47(7):1-8, DOI 10.1029/2020GL087464

Jongman B, Hochrainer-Stigler S, Feyen L, Aerts JC, Mechler R, Botzen WW, Bouwer LM, Pflug G, Rojas R, Ward PJ (2014) Increasing stress on disaster-risk finance due to large floods. Nature Climate Change $4(4): 264-268$

Donges JF, Zou Y, Marwan N, Kurths J (2009) The backbone of the climate network. EPL (Europhysics Letters) 87(4):48,007, DOI 10.1209/ 0295-5075/87/48007, URL https://doi.org/10. 1209/0295-5075/87/48007

Fan J, Meng J, Ludescher J, Chen X, Ashkenazy Y, Kurths J, Havlin S, Schellnhuber HJ (2021) Statistical physics approaches to the complex earth system. Physics Reports 896:1-84, DOI https://doi.org/10.1016/j.physrep.2020.09.005,

URL https://www.sciencedirect.com/science/ article/pii/S0370157320303458, statistical physics approaches to the complex Earth system

Tsonis A, Roebber P (2004) The architecture of the climate network. Physica A: Statistical Mechanics and its Applications 333:497-504, DOI https://doi.org/10.1016/j.physa.2003.10.045, URL https://www.sciencedirect.com/science/ URL https://agupubs . onlinelibrary . ical Research Letters 47(15):e2020GL088,185, DOI https://doi.org/10.1029/2020GL088185, URL https://agupubs.onlinelibrary.wiley.com/ https://journals . ametsoc.org/view/journals/

Mondal S, Mishra AK, Leung LR (2020) Spatiotemporal characteristics and propagation of summer extreme precipitation events over united states: A complex network analysis. GeophysPearson D, Fajman P (2020) A hydrometeoro-
logical assessment of the historic 2019 flood of nebraska, iowa, and south dakota. Bulletin of

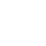

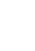


article/pii/S0378437103009646

${ }_{690}$ Quian Quiroga R, Kraskov A, Kreuz T, Grassberger P (2002) Performance of different synchronization measures in real data: A case study on electroencephalographic signals. Phys Rev E 65:041,903, DOI 10.1103/PhysRevE.65.041903, URL https://link. aps.org/doi/10.1103/PhysRevE.65.041903

Malik N, Bookhagen B, Marwan N, Kurths J (2011) Analysis of spatial and temporal extreme monsoonal rainfall over south asia using complex networks. Climate Dynamics 39:1-17, DOI 10.1007/ s00382-011-1156-4

Stolbova V, Martin P, Bookhagen B, Marwan N, Kurths J (2014) Topology and seasonal evolution of the network of extreme precipitation over the indian subcontinent and sri lanka. Nonlinear Processes in Geophysics 21(4):901-917, DOI 10.5194/ npg-21-901-2014, URL https://npg.copernicus. org/articles/21/901/2014/

Ozturk U, Malik N, Cheung K, Marwan N, Kurths J (2019) A network-based comparative study of extreme tropical and frontal storm rainfall over Japan. Climate Dynamics 53(1-2):521-532, DOI 10.1007/ s00382-018-4597-1

Mondal S, Mishra AK (2021) Complex networks reveal heatwave patterns and propagations over the usa. Geophysical Research Letters 48(2):e2020GL090,411, DOI https://doi.org/10.1029/2020GL090411, URL https://agupubs.onlinelibrary.wiley.com/ doi/abs/10.1029/2020GL090411, e2020GL090411 2020GL090411, https://agupubs . onlinelibrary . wiley.com/doi/pdf/10.1029/2020GL090411

Boers N, Bookhagen B, Marwan N, Kurths J, Marengo J (2013) Complex networks identify spatial patterns of extreme rainfall events of the south american monsoon system. Geophysical Research Letters 40(16):4386-4392, DOI https://doi.org/10.1002/ grl.50681, URL https://agupubs.onlinelibrary . wiley.com/doi/abs/10.1002/grl.50681

Boers N, Donner R, Bookhagen B, Kurths J (2014a) Complex network analysis helps to identify impacts of the el niño southern oscillation on moisture divergence in south america. Climate Dynamics 45:1-14, DOI 10.1007/s00382-014-2265-7

Boers N, Rheinwalt A, Bookhagen B, Barbosa HMJ, Marwan N, Marengo J, Kurths J (2014b) The south american rainfall dipole: A complex network analysis of extreme events. Geophysical Research Letters 41(20):7397-7405, DOI https://doi.org/10.1002/2014GL061829, URL https://agupubs.onlinelibrary. wiley.com/doi/abs/10.1002/2014GL061829, https://agupubs.onlinelibrary.wiley.com/
doi/pdf/10.1002/2014GL061829

Boers N, Goswami B, Rheinwalt A, Bookhagen B, Hoskins B, Kurths J (2019) Complex networks reveal global pattern of extreme-rainfall teleconnections. Nature 566(7744):373-377, DOI 10.1038/ s41586-018-0872-x

Konapala G, Mishra A (2017) Review of complex networks application in hydroclimatic extremes with an implementation to characterize spatio-temporal drought propagation in continental usa. Journal of Hydrology 555:600-620, DOI https://doi.org/10.1016/j.jhydrol.2017.10.033, URL https://www.sciencedirect.com/science/ article/pii/S0022169417307096

Agarwal A, Marwan N, Rathinasamy M, Merz B, Kurths J (2017) Multi-scale event synchronization analysis for unravelling climate processes: a wavelet-based approach. Nonlinear Processes in Geophysics 24(4):599-611, DOI 10.5194/ npg-24-599-2017, URL https://npg.copernicus. org/articles/24/599/2017/

Ciemer C, Boers N, Barbosa H, Kurths J, Rammig A (2018) Temporal evolution of the spatial covariability of rainfall in south america. Climate Dynamics 51, DOI 10.1007/s00382-017-3929-x

Victor JD, Purpura KP (1997) Metric-space analysis of spike trains: theory, algorithms and application. Network: Computation in Neural Systems 8(2):127164

Hirata Y, Aihara K (2009) Representing spike trains using constant sampling intervals. Journal of Neuroscience Methods 183(2):277 - 286, DOI https://doi.org/10.1016/j.jneumeth.2009.06.030,

URL http://www.sciencedirect.com/science/ article/pii/S0165027009003513

Suzuki S, Hirata Y, Aihara K (2010) Definition of distance for marked point process data and its application to recurrence plot-based analysis of exchange tick data of foreign currencies. International Journal of Bifurcation and Chaos 20(11):3699 3708, DOI 10.1142/S0218127410027970, URL https ://doi .org/10.1142/S0218127410027970, https://doi.org/10.1142/S0218127410027970

Ozken I, Eroglu D, Stemler T, Marwan N, Bagci GB, Kurths J (2015) Transformation-cost time-series method for analyzing irregularly sampled data. Physical Review E 91:062,911, DOI 10.1103/PhysRevE. 91.062911

Ozken I, Eroglu D, Breitenbach SFM, Marwan N, Tan L, Tirnakli U, Kurths J (2018) Recurrence plot analysis of irregularly sampled data. Physical Review E 98:052,215, DOI 10.1103/PhysRevE.98.052215 
Banerjee A, Goswami B, Hirata Y, Eroglu D, Merz B, Kurths J, Marwan N (2021) Recurrence analysis of extreme event-like data. Nonlinear Processes in Geophysics 28(2):213-229, DOI 10.5194/ npg-28-213-2021, URL https://npg.copernicus. org/articles/28/213/2021/

Hersbach H, Bell B, Berrisford P, Hirahara S, Horányi A, Muñoz-Sabater J, Nicolas J, Peubey C, Radu R, Schepers D, Simmons A, Soci C, Abdalla S, Abellan X, Balsamo G, Bechtold P, Biavati G, Bidlot J, Bonavita M, De Chiara G, Dahlgren P, Dee D, Diamantakis M, Dragani R, Flemming J, Forbes R, Fuentes M, Geer A, Haimberger L, Healy S, Hogan RJ, Hólm E, Janisková M, Keeley S, Laloyaux P, Lopez P, Lupu C, Radnoti G, de Rosnay P, Rozum I, Vamborg F, Villaume S, Thépaut JN (2020) The era5 global reanalysis. Quarterly Journal of the Royal Meteorological Society 146(730):1999-2049, DOI https://doi.org/10.1002/qj.3803, URL https: //rmets.onlinelibrary.wiley.com/doi/abs/10. 1002/qj.3803, https://rmets.onlinelibrary. wiley.com/doi/pdf/10.1002/qj. 3803

Hassler B, Lauer A (2021) Comparison of reanalysis and observational precipitation datasets including era5 and wfde5. Atmosphere 12(11), DOI 10.3390/atmos12111462, URL https://www.mdpi. com/2073-4433/12/11/1462

Japan Meteorological Agency, Japan (2013) Jra-55: Japanese 55-year reanalysis, daily 3-hourly and 6-hourly data. URL https://doi.org/10.5065/ D6HH6H41

Sivakumar B, Woldemeskel FM (2014) Complex networks for streamflow dynamics. Hydrology and Earth System Sciences 18(11):4565-4578, DOI 10.5194/hess-18-4565-2014, URL https://hess. copernicus .org/articles/18/4565/2014/

Wiedermann M, Donges JF, Kurths J, Donner RV (2017) Mapping and discrimination of networks in the complexity-entropy plane. Phys Rev E 96:042,304, DOI 10.1103/PhysRevE.96.042304, URL https ://link.aps.org/doi/10.1103/PhysRevE. 96.042304

Donges JF, Zou Y, Marwan N, Kurths J (2009) Complex networks in climate dynamics. The European Physical Journal Special Topics 174(1):157-179

Tsonis AA, Swanson KL, Wang G (2008) On the role of atmospheric teleconnections in climate. Journal of Climate 21(12):2990 3001, DOI 10.1175/2007JCLI1907.1, URL https://journals.ametsoc.org/view/journals/ clim/21/12/2007jcli1907.1.xml

${ }_{845}$ Radebach A, Donner RV, Runge J, Donges JF, Kurths J (2013) Disentangling different types of el niño episodes by evolving climate network analysis. Physical Review E 88(5):052,807

Agarwal A, Caesar L, Marwan N, Maheswaran R, Merz B, Kurths J (2019) Network-based identification and characterization of teleconnections on different scales. Scientific Reports 9

Newman MEJ (2010) Networks: An Introduction. Oxford University Press, DOI 10.1093/ACPROF:OSO/ 9780199206650.001.0001, URL https://doi.org/ 10.1093/ACPROF : OSO/9780199206650.001.0001

Freeman LC (1978) Centrality in social networks conceptual clarification. Social Networks 1(3):215-239, DOI https://doi.org/10.1016/0378-8733(78)90021-7, URL https://www.sciencedirect.com/science/ article/pii/0378873378900217

Golbeck J (2015) Chapter 21 - analyzing networks. In: Golbeck J (ed) Introduction to Social Media Investigation, Syngress, Boston, pp 221-235, DOI https: //doi.org/10.1016/B978-0-12-801656-5.00021-4, URL https://www.sciencedirect.com/science/ article/pii/B9780128016565000214

Molkenthin N, Rehfeld K, Marwan N, Kurths J (2014) Networks from flows - from dynamics to topology. Scientific Reports 4(4119), DOI 10.1038/srep04119, URL http://www . nature.com/srep/2014/140218/ srep04119/full/srep04119.html

Tupikina L, Molkenthin N, López C, HernándezGarcía E, Marwan N, Kurths J (2016) Correlation Networks from Flows. The Case of Forced and Time-Dependent Advection-Diffusion Dynamics. PLoS ONE 11(4):e0153,703, DOI 10.1371/journal. pone.0153703

Rheinwalt A, Marwan N, Kurths J, Werner P, Gerstengarbe FW (2012) Boundary effects in network measures of spatially embedded networks. EPL (Europhysics Letters), abstract $=$ In studies of spatially confined networks, network measures can lead to false conclusions since most measures are boundary affected This is especially the case if boundaries are artificial and not inherent in the underlying system of interest (eg, borders of countries) An analytical estimation of boundary effects is not trivial due to the complexity of measures The straightforward approach we propose here is to use surrogate networks that provide estimates of boundary effects in graph statistics This is achieved by using spatially embedded random networks as surrogates that have approximately the same link probability as a function of spatial link lengths The potential of our approach is demonstrated for an analysis of spatial patterns in characteristics of regional climate networks As an example networks derived from daily rainfall data and restricted to the re- 
gion of Germany are considered 100(2):28,002, DOI 10.1209/0295-5075/100/28002, URL https://doi. org/10.1209/0295-5075/100/28002

Barnett L, Di Paolo E, Bullock S (2007) Spatially embedded random networks. Phys Rev E 76:056,115, DOI 10.1103/PhysRevE.76.056115, URL https:// link.aps.org/doi/10.1103/PhysRevE.76.056115

Dettinger MD (2013) Atmospheric rivers as drought busters on the u.s. west coast. Journal of Hydrometeorology 14(6):1721 1732, DOI 10.1175/JHM-D-13-02.1, URL https://journals.ametsoc.org/view/journals/ hydr/14/6/jhm-d-13-02_1.xml

Xiong Y, Ren X (2021) Influences of atmospheric rivers on north pacific winter precipitation: Climatology and dependence on enso condition. Journal of Climate 34(1):277 - 292, DOI 10.1175/JCLI-D-20-0301.1, URL https://journals.ametsoc.org/view/journals/ clim/34/1/jcliD200301.xml

${ }_{920} \mathrm{Hu} \mathrm{H}$, Dominguez F, Wang Z, Lavers DA, Zhang G, Ralph FM (2017) Linking atmospheric river hydrological impacts on the u.s. west coast to rossby wave breaking. Journal of Climate 30(9):3381 - 3399, DOI 10.1175/JCLI-D-16-0386.1, URL https://journals.ametsoc.org/view/journals/ clim/30/9/jcli-d-16-0386.1.xml

Gonzales KR, Swain DL, Nardi KM, Barnes EA, Diffenbaugh NS (2019) Recent warming of landfalling atmospheric rivers along the west coast of the united states. Journal of Geophysical Research: Atmospheres 124(13):6810-6826, DOI https://doi.org/ 10.1029/2018JD029860

Woodruff J, Irish J, Camargo S (2013) Coastal flooding by tropical cyclones and sea-level rise. Nature 504:4452, DOI 10.1038/nature12855

Zhang Y, Wang K (2021) Global precipitation system size. Environmental Research Letters 16(5):054,005, DOI 10.1088/1748-9326/abf394, URL https: //doi. org/10.1088/1748-9326/abf 394

940 Gimeno L, Dominguez F, Nieto R, Trigo R, Drumond A, Reason CJ, Taschetto AS, Ramos AM, Kumar R, Marengo J (2016) Major mechanisms of atmospheric moisture transport and their role in extreme precipitation events. Annual Review of Environment and Resources 41(1):117-141, DOI 10.1146/annurev-environ-110615-085558, URL https://doi.org/10.1146/ annurev-environ-110615-085558, https://doi. org/10.1146/annurev-environ-110615-085558

${ }_{950}$ Guan B, Waliser DE (2015) Detection of atmospheric rivers: Evaluation and application of an algorithm for global studies. Journal of Geophysical
Research: Atmospheres 120(24):12,514-12,535, DOI https://doi.org/10.1002/2015JD024257, URL https://agupubs .onlinelibrary. wiley.com/doi/abs/10.1002/2015JD024257, https://agupubs.onlinelibrary.wiley.com/ doi/pdf/10.1002/2015JD024257

Ralph FM, Rutz JJ, Cordeira JM, Dettinger M, Anderson M, Reynolds D, Schick LJ, Smallcomb C (2019) A scale to characterize the strength and impacts of atmospheric rivers. Bulletin of the American Meteorological Society 100(2):269 - 289, DOI 10.1175/BAMS-D-18-0023.1, URL https://journals.ametsoc.org/view/journals/ bams $/ 100 / 2 /$ bams-d-18-0023.1.xml

Barlow M, Gutowski W, Gyakum J, Katz R, Lim Y, Schumacher R, Wehner M, Agel L, Bosilovich M, Collow A, Gershunov A, Grotjahn R, Leung R, Milrad S, Min S (2019) North american extreme precipitation events and related large-scale meteorological patterns: a review of statistical methods, dynamics, modeling, and trends. Climate Dynamics 53:6835 6875

Concannon P, Brooks H, Doswell III C (2000) Climatological risk of strong and violent tornadoes in the united states

Bluestein HB (2006) Tornado alley: monster storms of the Great Plains. Oxford University Press, USA

Lukens KE, Berbery EH, Hodges KI (2018) The imprint of strong-storm tracks on winter weather in north america. Journal of Climate 31(5):2057 - 2074, DOI 10.1175/JCLI-D-17-0420.1, URL https://journals . ametsoc.org/view/journals/ clim/31/5/jcli-d-17-0420.1.xml

Chang EKM (2009) Diabatic and orographic forcing of northern winter stationary waves and storm tracks. Journal of Climate 22(3):670 - 688, DOI 10.1175/2008JCLI2403.1, URL https://journals.ametsoc.org/view/journals/ clim/22/3/2008jcli2403.1.xml

Brayshaw DJ, Hoskins B, Blackburn M (2009) The basic ingredients of the north atlantic storm track. part i: Land?sea contrast and orography. Journal of the Atmospheric Sciences 66(9):2539 $2558, \quad$ DOI 10.1175/2009JAS3078.1, URL https://journals.ametsoc.org/view/journals/ atsc/66/9/2009jas3078.1.xml

Colucci SJ (1976) winter cyclone frequencies over the eastern united states and adjacent western atlantic, 1000 1964?1973: Student paper?first place winner of the father james b. macelwane annual award in meteorology, announced at the annual meeting of the ams, philadelphia, pa., 21 january 1976. Bulletin of the American Meteorological Society 57(5):548 
- 553, DOI 10.1175/1520-0477(1976)057〈0548: WCFOTE $>2.0 . C O ; 2$, URL https://journals. ametsoc.org/view/journals/bams/57/5/

1520-0477_1976_057_0548_wcfote_2_0_co_2.xml

Complex network approach for detecting tropical cyclones. Climate Dynamics DOI 10.1007/ s00382-021-05871-0

Wolf F, Bauer J, Boers N, Donner RV (2020) Event synchrony measures for functional climate network analysis: A case study on south american rainfall dynamics. Chaos: An Interdisciplinary Journal of Nonlinear Science 30(3):033,102, DOI 10. 1063/1.5134012, URL https://doi.org/10.1063/ 1.5134012, https://doi.org/10.1063/1.5134012 